\title{
Exploring non-stationarity patterns in schizophrenia: neural reorganization abnormalities in the alpha band
}

\author{
Pablo Núñez ${ }^{1}$, Jesús Poza ${ }^{1,2,3}$, Alejandro Bachiller ${ }^{1}$, Javier Gomez-Pilar ${ }^{1}$, Alba Lubeiro ${ }^{4}$, \\ Vicente Molina $^{3,4,5}$, and Roberto Hornero ${ }^{1,2,3}$ \\ ${ }^{1}$ Biomedical Engineering Group, University of Valladolid, Valladolid, Spain \\ ${ }^{2}$ IMUVA, Mathematics Research Institute, University of Valladolid, Valladolid, Spain \\ ${ }^{3}$ INCYL, Institute of Neurosciences of Castilla y León, University of Salamanca, Salamanca, \\ Spain \\ ${ }^{4}$ Department of Psychiatry, Faculty of Medicine, University of Valladolid, Valladolid, Spain \\ ${ }^{5}$ Hospital Clínico Universitario, Valladolid, Spain
}

Email: pablo.nunez@gib.tel.uva.es (Pablo Núñez)

\begin{abstract}
Objective. The aim of this paper was to characterize brain non-stationarity during an auditory oddball task in schizophrenia ( $\mathrm{SCH})$. The level of non-stationarity was measured in the baseline and response windows of relevant tones in SCH patients and healthy controls. Approach. Eventrelated potentials were recorded from $28 \mathrm{SCH}$ patients and 51 controls. Non-stationarity was estimated in the conventional electroencephalography frequency bands by means of KullbackLeibler divergence (KLD). Relative power (RP) was also computed to assess a possible complementarity with KLD. Main results. Results showed a widespread statistically significant increase in the level of non-stationarity from baseline to response in all frequency bands for both groups. Statistically significant differences in non-stationarity were found between SCH patients and controls in beta-2 and especially in the alpha band. SCH patients showed more nonstationarity in the left parieto-occipital region during the baseline window in the beta-2 band. A leave-one-out cross validation classification study with feature selection based on binary stepwise logistic regression to discriminate between $\mathrm{SCH}$ patients and controls provided an accuracy of $89.87 \%$ and area under ROC of 0.9510. Significance. KLD can characterize transient neural reorganization during an attentional task in response to novelty and relevance. Our findings suggest anomalous reorganization of neural dynamics in SCH during an oddball task. The abnormal frequency-dependent modulation found in $\mathrm{SCH}$ patients during relevant tones is in agreement with the hypothesis of aberrant salience detection in SCH. The increase in nonstationarity in the alpha band during the active task supports the notion that this band is involved in top-down processing. The baseline differences in the beta- 2 band suggest that hyperactivation of the default mode network during attention tasks may be related to SCH symptoms. Furthermore, the binary stepwise logistic regression procedure selected features from both KLD and RP, supporting the idea that these measures can be complementary.
\end{abstract}

PACS: $\quad$ 87.19.L Neuroscience

87.19.le EEG and MEG

87.85.dq Neural networks

87.85.Ng Biological signal processing

89.70.Cf Entropy and other measures of information

Submitted to: Journal of Neural Engineering

\section{Introduction}

Schizophrenia $(\mathrm{SCH})$ is characterized by a cluster of symptoms and signs that differ among subjects, including, among others, hallucinations and delusions, reduced motivation and impairment in cognitive processing [1]. SCH has an incidence of 15.2/100,000 persons per year [2], and a significant contribution to the global burden of disease [1]. SCH commonly has its onset in early adulthood and approximately two-thirds of affected individuals have persisting 
symptoms even after optimal treatment [1]. The associated SCH costs are high for national healthcare services as well as for patients, their families and caregivers [3]. Employment difficulties are common among patients, substantial burden is reported on their families and mortality rates are high [3].

Electroencephalography (EEG) recordings have been used to analyze neural processing in SCH through the analysis of event-related potentials (ERPs) [4-7]. EEG captures the summation of neural oscillations in the brain and their synchronization [8]. This information is used to gain insight into the pathophysiological processes in neuropsychiatric disorders [4]. Early on, research of aberrant oscillatory activity in SCH focused on resting-state EEG data. Recent studies have complemented this framework with task-related SCH research, which may provide further insights into dysfunctional cognitive processes and impaired oscillatory activity in $\mathrm{SCH}[8]$.

Event-related EEG analysis has been usually carried out in either the time or the frequency domain. The time-domain approach involves averaging a set of trials or epochs, time and phaselocked to a certain event, producing a single ERP at each channel [9]. The frequency-domain approach averages changes in the frequency power spectrum of the whole EEG data timelocked but not phase-locked to the same events, which outputs a two-dimensional image called the event-related spectral perturbation [9]. Neither method is capable of fully modeling brain dynamics. Time-frequency analyses overcome several limitations of traditional ERP analyses, such as the lack of simultaneous time and spectral information. They comprise a variety of methods that capture different aspects of magnitude and phase of EEG oscillations [4]. Furthermore, time-frequency estimation does not assume stationarity [10]. Traditional ERP analyses lack the ability to view the brain as a parallel processing of information. It has been shown that oscillations at various frequencies reflect distinct neural processes that co-occur and interact [4] and that phase and power measures are more sensitive to $\mathrm{SCH}$ than traditional ERP components such as the P300 [11]. Time-frequency methods usually follow the single trial approach to ERP analysis. This analysis is useful to jointly analyze the evoked and induced response, of which the latter is not phase-locked to the stimulus onset and is suppressed when trials are averaged [4]. In single trial analysis, measures are calculated for each artifact-free trial and then averaged across trials, as opposed to calculating measures for the already averaged trials [4]. This prevents oscillations that are out of phase with respect the stimulus from being lost in the averaging process.

The auditory oddball paradigm is used to study neural response to novelty and relevance. A common implementation is composed of standard, distractor and target tones. Distractor tones usually have the same frequency of occurrence as target tones. As subjects are asked to respond to target tones, distractor tones only possess a novelty component, while target tones are associated to both novelty and relevance [12]. Several methods have been used to analyze transient dynamics in neural activity during an auditory oddball task or during resting-state. Such measures, of a time-dependent nature, include wavelet entropy $[6,13]$, multiscale entropy [14], median frequency [12,15] and relative power (RP) [15], among others. Many of these studies have found widespread lower neural reorganization (i.e., transient dynamical reorganization of brain activity during an attentional process) for $\mathrm{SCH}$ subjects compared to controls during the active task response $[6,12,15]$.

A strictly non-stationary process is a stochastic process whose joint probability distribution changes over time [16]. The formal concept of strict stationarity is very rigorous, as it implies that all moments of all degrees are constant [17]. On the other hand, weak stationarity means that the mean and variance of a stochastic process are constant and the autocovariance, and thus the autocorrelation, only depend on the time difference between two time points [17]. This concept is closely related to the power spectral density (PSD), since the PSD can be computed as the Fourier transform of the autocorrelation of a signal [18]. Thereby, frequency components do not change with time in a stationary process [19]. The short-time Fourier transform is a method of signal analysis that is applicable to non-stationary signals, as it divides them into time windows over which stationarity can be assumed. Furthermore, the wavelet transform can 
be considered an extension of the short-time Fourier transform [20]: it is clear then that there is a link between the PSD and the wavelet transform and, therefore, between the wavelet transform and the concept of stationarity.

The spectral content of ERPs displays changes in the magnitude and phase of EEG oscillations at specific frequencies, as well as transient phase resetting of the ongoing EEG [4], which makes for highly variable signals. It is therefore interesting to measure the level of nonstationarity of the EEG signals recorded during an auditory oddball task, as traditional measures are based on the assumption that the EEG is a pseudo-stationary signal [16]. There are different measures of non-stationarity, such as spectral entropy or the degree of stationarity [19]. In a preliminary work, the latter was used to analyze non-stationarity in SCH and statistically significant differences between the baseline and response windows were found for both SCH patients and controls [21]. Hence, we posed the hypothesis that a measure of the nonstationarity for each time and/or frequency could provide further insight into brain dynamics. Kullback-Leibler divergence (KLD) is an information theory measure that can characterize the non-stationarity of a signal by computing the divergence between a time-frequency representation (TFR) and a uniform distribution [19]. However, one of the advantages of KLD over other measures of non-stationarity is that it allows the comparison of a signal with any distribution [19]. KLD has been recommended over other distance and divergence measures as it satisfies certain mathematical properties, particularly the Pinsker-Csiszár inequality, which bounds the statistical distance between two probability distributions in terms of the KLD $[22,23]$. It was previously applied to EEG recordings of patients with epilepsy and it was found that abnormal frequency patterns corresponding to epileptic seizures were better identified with KLD than with Shannon entropy [24]. Thus, a low value of KLD means that the two variables are close in distribution. Furthermore, in contrast to some of the previously mentioned classical measures, the proposed KLD implementation is frequency-dependent, which enables the analysis of each frequency band separately. An additional parameter, RP, was computed as well in order to explore its possible complementarity with KLD. RP could also be related to the level of non-stationarity, as it has been used to assess the proportion of total spectral power that can be attributed to a single band [15].

In this study, we hypothesized that event-related neural reorganization during an attentional process could be reflected in increased levels of non-stationarity in the brain during target trials in an auditory oddball task, and that measuring the level of non-stationary transient changes in an EEG recording could be one way to quantify the reorganization of brain activity. The aim of this study was three-fold: (i) to characterize neural reorganization during an auditory oddball task by measuring the non-stationarity with KLD; (ii) to explore group-level differences in nonstationarity levels between SCH patients and controls; and (iii) to evaluate whether KLD and $\mathrm{RP}$ could be useful to discriminate between $\mathrm{SCH}$ patients and controls.

\section{Subjects and Signals}

\subsection{Subjects}

Seventy-nine subjects agreed to participate in this study. Of these, 28 were SCH patients (13 men and 15 women; $31.19 \pm 10.43$ years, mean \pm standard deviation, SD) and 51 healthy controls ( 24 men and 27 women; $29.31 \pm 9.74$ years, mean \pm SD). Non-significant differences were found in age $(U=564.000, p=0.412$, Mann-Whitney $U$-test $)$ and gender $\left(\chi^{2}=0.168\right.$, $p=0.682$, Chi-squared test). SCH patients were diagnosed according to the Diagnostic and Statistical Manual of Mental Disorders $5^{\text {th }}$ edition (DSM-V) [25]. In order to discard controls with a major psychiatric history, subjects were interviewed beforehand. The exclusion criteria can be summarized as follows: (i) any previous neurological illness or major head trauma that resulted in a loss of consciousness longer than one minute; (ii) electroconvulsive therapy; (iii) previous or current substance abuse, nicotine and caffeine excluded; (iv) for patients only, any other psychiatric process different from $\mathrm{SCH}$; (v) for controls, past or present psychiatric diagnosis or current treatment with drugs known to act on the nervous system. Written informed consent was obtained from SCH patients and healthy controls, after full written information was 
provided. The ethics and research board of the 'Hospital Clínico Universitario' of Valladolid (Spain) endorsed the study according to The Code of Ethics of the World Medical Association (Declaration of Helsinki).

\subsection{EEG Recordings}

EEG recordings were acquired with a BrainVision ${ }^{\circledR}$ (Brain Products GmbH; Munich, Germany) system, consisting of 32 sensors mounted in an electrode cap (Electro-Cap International, Inc.; Eaton, Ohio, USA) and placed according to the International 10/10 System. Thirteen minutelength recordings were acquired at a sampling rate of $500 \mathrm{~Hz}$. Participants underwent a threestimulus auditory oddball task. Tones (duration $50 \mathrm{~ms}$, rise and fall time $5 \mathrm{~ms}$, intensity $90 \mathrm{~dB}$ and inter-stimulus interval randomly ranged between 1.16-1.40 s [6]) were presented to participants in random 600 tone series. These consisted of target $(500 \mathrm{~Hz})$, distractor $(1000 \mathrm{~Hz})$, and standard $(2000 \mathrm{~Hz})$ tones, with probabilities of $0.20,0.20$, and 0.60 respectively. Subjects were asked to press a button whenever they heard a target tone. Unattended target tones were discarded (target tones not followed by a button press).

Channels TP9 and TP10 were discarded due to abundant muscle artifacts throughout most recordings. It is well documented that EEG recordings are prone to the propagation of muscle artifacts [26]. In order to assure that the remaining channels were free from contamination, the recordings were carefully inspected by a specialist and no other channels were found to be affected by artifacts throughout. Recordings were referenced over $\mathrm{Cz}$ electrode and rereferenced to the average activity of all active sensors [27], yielding a total of 31 channels. Electrode impedance was kept under $5 \mathrm{k} \Omega$. Then, each ERP recording was filtered with a 1-70 $\mathrm{Hz}$ finite impulse response (FIR) filter and a $50 \mathrm{~Hz}$ notch filter. Afterwards, a three-step artifact rejection procedure was conducted to discard the remaining noisy segments. Firstly, an independent component analysis (ICA) was carried out to decompose ERPs into 31 components; then, after visual inspection, ICA components associated with artifacts such as eye blinks were discarded. Secondly, data were reconstructed and segmented into $1000 \mathrm{~ms}$ ERP trials (-300 ms to $700 \mathrm{~ms}$ with respect to the stimulus onset, 500 samples per epoch). Finally, artifacts were automatically rejected by means of an adaptive thresholding method in order to remove ERP trials displaying amplitudes that exceeded a statistically based local threshold. In a first step, the mean and standard deviation of each channel for each stimulus type (standard, distractor and target) was computed. In a second step, for each stimulus type, trials that exceeded mean $\pm 4 \times$ standard deviation in at least two channels were discarded [7].

The number of total trials (mean $\pm \mathrm{SD}$ ) for standard condition was $400.62 \pm 10.06$ for controls $(295.92 \pm 32.31$ selected after artifact rejection) and $400.85 \pm 11.73$ for patients ( $288.75 \pm 37.00$ selected after artifact rejection). In the case of distractor trials, the total number for controls was $100.64 \pm 7.51$ (72.76 \pm 8.81 selected after artifact rejection) and for patients $99.42 \pm 7.16$ (65.42 \pm 15.94 selected after artifact rejection). The number of trials for target condition was $97.07 \pm 9.58$ for controls $(70.86 \pm 11.86$ selected after artifact rejection) and $87.07 \pm 17.19$ for patients $(62.57 \pm 16.26$ selected after artifact rejection). The percentage of attended target trials for each group was $98.39 \% \pm 2.12 \%$ for controls and $88.20 \% \pm 17.27 \%$ for SCH patients. The reaction times were $242.43 \pm 33.05 \mathrm{~ms}$ for controls and $277.15 \pm$ $51.30 \mathrm{~ms}$ for $\mathrm{SCH}$ patients.

\section{Methods}

\subsection{Continuous wavelet transform}

There are event-related changes in the magnitude and phase of EEG oscillations at certain frequencies [4]. Time-frequency analysis can provide additional information not apparent in an ongoing EEG, and comprises many methods and measures that seek to capture different aspects of EEG magnitude and phase at specific frequencies [4]. In this paper, the continuous wavelet transform (CWT) was used to estimate the TFR. CWT is conceptually related to the short-term Fourier transform [20]. However, it is better suited for ERP study, since it provides a good compromise between time and frequency resolutions [28]. Wavelet analysis allows flexible 
control over the resolution with which neuroelectric components and events can be localized in time and frequency. This control translates into better detection of dynamic ERP components [29].

In order to correctly model biological signals, such as EEG, the waveforms in the wavelet must provide a biologically plausible fit to the signal being modelled [4]. In this study, the Morlet wavelet was used. Two of its parameters (center frequency $\Omega_{c}$ and bandwidth parameter $\left.\Omega_{b}\right)$ need to be adjusted. In order to obtain a good balance between the time $(\Delta t)$ and frequency $(\Delta f)$ resolution at low frequencies both parameters were set to 1 [7].

Heisenberg boxes represent the tradeoff between temporal and frequency resolutions in the CWT and are defined as rectangles with constant area and whose width depends on the timefrequency resolution [30]. In this study, the width of the Heisenberg box was chosen to be two times $\Delta t$ and $\Delta f$ [7]. The average $\Delta t$ and $\Delta f$ values for each band are shown in table 1 .

ERP signals are finite-length time recordings. This results in errors at the beginning and end of the wavelet power spectrum. In order to overcome this limitation, zero padding is introduced at the beginning and end of the ERP recordings, but this introduces discontinuities at the edges. The cone of influence (COI) is the region of the wavelet spectrum in which edge effects can be ignored [31], avoiding the distortions introduced by the zero padding.

\subsection{Kullback-Leibler Divergence}

The term 'non-stationarity' refers to the characteristics of a time series, such as mean, variance and spectral characteristics changing with time [16]. The neural system has intrinsically timevarying properties [19]. A variety of statistical tests have revealed different estimates of the amount of time during which EEG can be considered stationary, ranging from seconds to minutes [16]. KLD is a metric that measures the discrepancy between two distributions $p_{i}$ and $q_{i}$ [19]. The marginal frequency distribution $\operatorname{tf} r\left(f_{i}\right)$ and the temporal TFR distribution are described in equations (1) and (2) respectively, while KLD is described in equation (3) [19]:

$$
\begin{gathered}
t f r\left(f_{i}\right)=\frac{1}{N} \sum_{n=1}^{n=N} t f r\left(f_{i}, n \Delta t\right), \\
p_{f_{i}, n \Delta t}=\frac{1}{N} \frac{t f r\left(f_{i}, n \Delta t\right)}{t f r\left(f_{i}\right)}, \\
K L D\left(f_{i}\right)=\sum_{n=1}^{n=N} q_{f_{i}, n \Delta t} \log _{2} \frac{q_{f_{i}, n \Delta t}}{p_{f_{i}, n \Delta t}},
\end{gathered}
$$

$\operatorname{tfr}\left(f_{i}, n \Delta t\right)$ is the TFR of the signal at each frequency and time point. In this study, $\operatorname{tfr}\left(f_{i}, n \Delta t\right)$ corresponds with the wavelet scalogram. For stationary signals, $t f r\left(f_{i}, n \Delta t\right)$ is constant at each $f_{i}$, that is, $\operatorname{tfr}\left(f_{i}\right)=\operatorname{tfr}\left(f_{i}, n \Delta t\right)$. In such a case, $K L D\left(f_{i}\right)$ is equal to zero. $K L D\left(f_{i}\right)$ increases as the signal becomes less stationary at a given frequency $f_{i}$. In our study, KLD was used to quantify how much the TFR differs from a uniform distribution in the time domain $\left\{q_{f i, n \Delta t}=q_{0}=1 / N\right\}$. Hence KLD can be interpreted as how far the TFR is from being completely stationary.

One second-length target trials were decomposed into the baseline, defined as the [-300 0] ms window (where 0 marks the stimulus onset), and the response [150 550] ms window [15]. Time-frequency analyses were computed separately for each of these windows and averaged in five frequency bands: theta $(\theta, 4-8 \mathrm{~Hz})$, alpha $(\alpha, 8-13 \mathrm{~Hz})$, beta- $1\left(\beta_{1}, 13-19 \mathrm{~Hz}\right)$, beta-2 $\left(\beta_{2}, 19-30 \mathrm{~Hz}\right)$ and gamma $(\gamma, 30-70 \mathrm{~Hz})$.

Table 1. Average $\Delta t$ and $\Delta f$ values for each band (mean \pm standard deviation).

\begin{tabular}{lccccc}
\cline { 2 - 6 } & Theta & Alpha & Beta-1 & Beta-2 & Gamma \\
\hline$\Delta t(\mathrm{~s})$ & $0.0875 \pm 0.0211$ & $0.0487 \pm 0.0079$ & $0.0317 \pm 0.0039$ & $0.0208 \pm 0.0029$ & $0.0105 \pm 0.0026$ \\
\hline$\Delta f(\mathrm{~Hz})$ & $0.9549 \pm 0.2179$ & $1.6711 \pm 0.2639$ & $2.5465 \pm 0.3099$ & $3.8993 \pm 0.5397$ & $7.9975 \pm 1.8492$ \\
\hline
\end{tabular}


The delta band was excluded from the analysis, as its associated wavelet duration is longer than hundreds of milliseconds. It therefore requires a window length longer than the baseline and response intervals to be correctly analyzed [7]. Figure 1 shows two sample scalograms with the baseline and response COI. The average KLD values at the Pz electrode for a SCH patient and a healthy control, for both the baseline and response time windows, are also shown.

\subsection{Relative Power}

$\mathrm{RP}$ is a measure commonly used to characterize the spectrum of a signal. It quantifies the relative contribution of different frequency bands to the global power spectrum and thus can be useful to evaluate changes in the spectral content of an EEG recording [15]. RP is independent of the measuring equipment and has relatively low inter-subject variability [32]. For each frequency band, RP is computed by summing the contribution of each spectral component in the band [15]:

$$
R P_{f_{p}}=\sum_{f \in f_{p}} P S D(f), \quad f_{p} \in\left\{\theta, \alpha, \beta_{1}, \beta_{2}, \gamma\right\} .
$$

In this study, we computed RP in order to study the spectral features of the EEG recordings, assess the validity of the database by comparing the results with those of a different one [15], and check its relationship with KLD. It is worth pointing out that the TFR needs to be normalized to the sum of the absolute values in all bands in order for RP to be a relative measure. In this study, the CWTs were normalized in the 1-70 Hz frequency range before the computation of both KLD and RP.

\subsection{Statistical and Classification Analyses}

For every artifact-free trial, KLD and RP were computed at each frequency band and electrode, in the baseline and response time windows. The COI was applied to the wavelets before computing the measures. After this, KLD and RP values were averaged across trials for every frequency band under study, reducing each subject's data to a single value for each channel, time window, and frequency band.

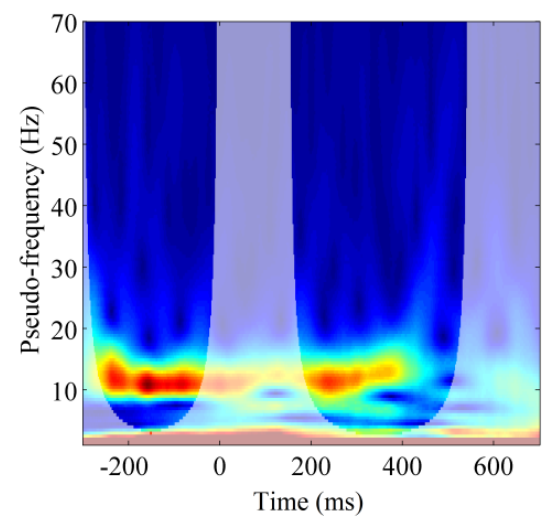

(a)

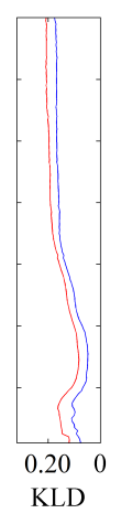

- Baseline - Response

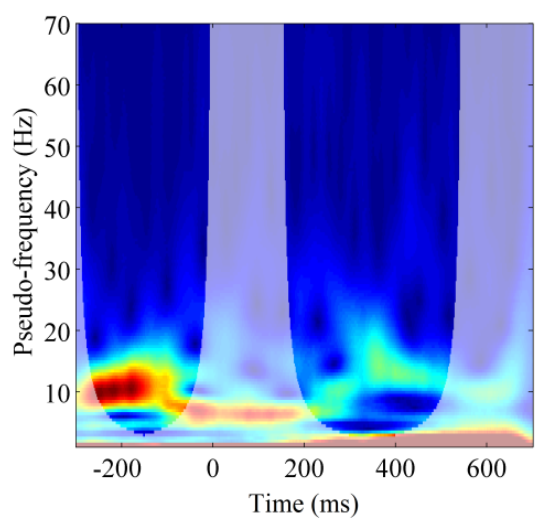

(b)

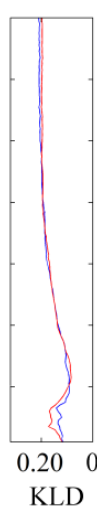

- Baseline - Response

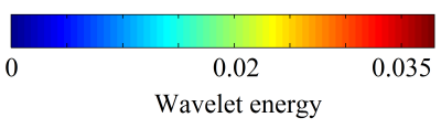

Figure 1. Averaged scalograms across trials at the Pz electrode for: (a) healthy control and (b) a SCH patient. The scalograms were computed by squaring the absolute value of the CWT coefficients. The COI, where edge effects can be ignored, is represented by a transparency mask overlaid over the scalogram. The COI is depicted for the baseline and response windows. The average KLD values across trials for each subject at the Pz electrode are displayed to the right of each scalogram. KLD was computed for the whole frequency band $(1-70 \mathrm{~Hz})$. The blue line represents the baseline values, while the red line corresponds to the response values. 
In order to capture event-related changes in brain activity, a baseline correction procedure was carried out by subtracting the baseline values from the response values at each electrode and frequency band $(\triangle \mathrm{KLD}, \Delta \mathrm{RP})[4] . \Delta \mathrm{KLD}$ values are positive when there is an increase from response to baseline in the level of non-stationarity and negative when there is a decrease.

An exploratory analysis was carried out to analyze the distribution of KLD, $\triangle K L D, R P$ and $\triangle \mathrm{RP}$ values. Then, grand-average KLD and RP values over all electrodes were analyzed using repeated measures ANOVA with 'group' (control, schizophrenic) as between-subject factor and 'frequency band' (theta, alpha, beta-1, beta-2, gamma), 'window' (baseline, response) and 'stimulus' (standard, distractor, target) as repeated measures factors. Greenhouse-Geisser correction was applied to adjust the degrees-of-freedom of the model whenever Mauchly's test indicated that the sphericity assumption was violated. Afterwards, 'group' by 'frequency band' by 'window' interactions for each stimulus type were examined to determine whether target tones obtained the more significant results. Dependent-sample $t$-tests were applied to each group to determine differences between baseline and response KLD values. In addition, differences between both groups in $\triangle \mathrm{KLD}$ were analyzed by means of univariate ANOVAs. Bonferroni correction was used to adjust the level of statistical significance for multiple testing $(\alpha=0.01)$.

In the case of the frequency bands that showed significant between-group effects, sensorlevel statistical analyses were carried out to evaluate spatial differences for $\triangle$ KLD by means of dependent-sample $t$-tests to compare baseline and response for each group and univariate ANOVAs to analyze between-group differences in the baseline and in the response window for target trials. In order to correct for multiple comparisons, a false discovery rate (FDR) controlling procedure was used [33]. Due to the high number of comparisons (31 EEG channels), FDR correction is preferred over Bonferroni, as the latter may be overly conservative and might overlook true effects [34].

Finally, $\triangle \mathrm{KLD}$ and $\triangle \mathrm{RP}$ classification performance was analyzed. The classification features were the average $\triangle \mathrm{KLD}$ and $\triangle \mathrm{RP}$ values across target trials at each electrode and frequency band, for a total of 155 features for each measure (31 channels $\times 5$ frequency bands). Four feature sets were computed: the first set of features (FS1) was built by simply averaging the $\triangle$ KLD values across channels, for a total of 5 features; the second feature set (FS2) included only $\triangle \mathrm{KLD}$ features for each channel; the third dataset (FS3) included only $\triangle \mathrm{RP}$ features for each channel; the last dataset (FS4) included both measures for each channel as possible features, in order to assess the complementarity of $\triangle \mathrm{KLD}$ and $\triangle \mathrm{RP}$. A feature selection procedure was performed to select the most suitable features for classification in datasets FS2, FS3 and FS4.

The features were selected using a binary stepwise logistic regression (LR) with a conditional forward selection approach. In order to prevent bias in the feature selection procedure, a leave-one-out cross validation (LOO-CV) procedure was performed: for each of the 79 folds of the CV, a LR feature selection was carried out. Afterwards, the 8 features that were selected the most during the $\mathrm{CV}$ were chosen for each dataset in order to prevent overfitting, as there were only 79 subjects in the study [35]. Another LOO-CV procedure was then performed to assess classification performance for each feature set after the appropriate features were selected. The classifier used in this final step was LR. Receiver operating characteristic (ROC) curves for the two feature sets were computed to assess classification robustness.

Signal processing was carried out using Matlab (version 2012.a, Mathworks, Natick, MA). Statistical analyses were carried out using SPSS Statistics (version 20, IBM Corp, Armonk, NY). 


\section{Results}

\subsection{Global analysis}

In a first step, KLD values were averaged over all electrodes to explore global patterns of statistically significant differences. The repeated measures ANOVA revealed significant interactions for the 'stimulus' $\left(F[1.758,135.329]=32.106, p<0.0001\right.$, partial $\eta^{2}=0.294$, Greenhouse-Geisser correction: $\varepsilon=0.879)$, 'frequency band' $(F[2.411,185.680]=722.334$, $p<0.0001$, partial $\eta^{2}=0.904$, Greenhouse-Geisser correction: $\varepsilon=0.603$ ) and 'window' factors $\left(F[1]=4838.516, p<0.0001\right.$, partial $\left.\eta^{2}=0.984\right)$.

Significant 'window' by 'group' $\left(F[1]=13.031, p<0.0001\right.$, partial $\left.\eta^{2}=0.145\right)$, 'stimulus' by 'window' $\left(F[1.748,134.572]=17.556, \quad p<0.0001\right.$, partial $\eta^{2}=0.186$, Greenhouse-Geisser correction: $\varepsilon=0.874)$, 'stimulus' by 'frequency band' $(F[4.481,345.029]=21.739, p<0.0001$, partial $\eta^{2}=0.220$, Greenhouse-Geisser correction: $\varepsilon=0.560$ ), 'frequency band' by 'window' $\left(F[2.568,197.752]=718.286, p<0.0001\right.$, partial $\eta^{2}=0.903$, Greenhouse-Geisser correction: $\varepsilon=0.642)$, 'stimulus' by 'frequency band' by 'window' $(F[4.789,386.736]=27.579, p<0.0001$, partial $\eta^{2}=0.264$, Greenhouse-Geisser correction: $\varepsilon=0.599$ ), 'group' by 'frequency band' by 'window' $\left(F[2.568,197.752]=9.323, \quad p<0.0001\right.$, partial $\eta^{2}=0.108$, Greenhouse-Geisser correction: $\varepsilon=0.642$ ) and 'group' by 'stimulus' by 'window' by 'frequency band' interactions $\left(F[4.789,368.736]=4.606, p=0.001\right.$, partial $\eta^{2}=0.056$, Greenhouse-Geisser correction: $\left.\varepsilon=0.599\right)$ were also observed.

For standard tones, a statistically significant 'group' by 'window' by 'frequency band' interaction was found $\left(F[2.731,210.280]=3.411, p=0.022\right.$, partial $\eta^{2}=0.042$, GreenhouseGeisser correction: $\varepsilon=0.683$ ). For distractor tones, a statistically significant 'group' by 'window' by 'frequency band' interaction was also found $(F[2.804,215.878]=4.083, p=0.009$, partial $\eta^{2}=0.050$, Greenhouse-Geisser correction: $\varepsilon=0.701$ ). Finally, for target tones, the 'group' by 'window' by 'frequency band' interaction was also statistically significant $(F[2.452$, 188.807] $=4.083, p<0.0001$, partial $\eta^{2}=0.121$, Greenhouse-Geisser correction: $\varepsilon=0.613$ ). It can be clearly seen that target tones displayed the most significant 'group' by 'window' by 'frequency band' interactions: for this reason all further tests were performed on target tones only.

Dependent-sample $t$-tests showed that both controls and $\mathrm{SCH}$ patients exhibited statistically significantly higher KLD values in the response than in the baseline window for all the frequency bands. Univariate ANOVAs showed that mean $\triangle \mathrm{KLD}$ values were statistically significantly lower for SCH patients than for controls in the alpha band and beta- 2 band. Nonsignificant effects were found in theta, beta- 1 and gamma. The results of both tests are displayed in table 2. Figure $3 \mathrm{a}$ shows the boxplots corresponding to the grand-average $\triangle \mathrm{KLD}$ values for controls and SCH patients for target tones.

In the case of RP, dependent-sample $t$-tests revealed that controls exhibited statistically significantly higher RP values in the response than in the baseline for theta, whereas the opposite behavior was observed for alpha, beta-1, beta-2 and gamma. Similar results were observed for SCH patients. RP values were statistically significantly higher in the response window when compared to the baseline for theta, whereas the opposite behavior was found for alpha and beta- 2 . 
Table 2. Results of the global analysis for KLD and $\triangle K L D$. Bonferroni corrected for 5 comparisons; i.e., each frequency band.

\begin{tabular}{ccccc|ccc}
\cline { 3 - 8 } & \multicolumn{4}{c|}{ KLD Response vs. Baseline } & \multicolumn{3}{c}{} \\
\multicolumn{4}{c}{ Controls } & \multicolumn{2}{c|}{ SCH } & \multicolumn{2}{c}{$\Delta$ KLD Controls vs. SCH patients } \\
& $t[50]$ & $p$-value & $t[27]$ & $p$-value & $F[1,77]$ & $p$-value & partial $\eta^{2}$ \\
\hline$\theta$ & 41.603 & $<0.0001$ & 33.792 & $<0.0001$ & 4.330 & 0.041 & 0.053 \\
$\alpha$ & 24.065 & $<0.0001$ & 12.195 & $<0.0001$ & 17.527 & $<0.0001$ & 0.185 \\
$\beta_{1}$ & 18.576 & $<0.0001$ & 13.563 & $<0.0001$ & 0.776 & 0.381 & 0.010 \\
$\beta_{2}$ & 20.276 & $<0.0001$ & 10.159 & $<0.0001$ & 11.317 & 0.001 & 0.128 \\
$\gamma$ & 13.521 & $<0.0001$ & 6.502 & $<0.0001$ & 2.072 & 0.154 & 0.026 \\
\hline
\end{tabular}

Table 3. Results of the global analysis for RP and $\triangle R P$. Bonferroni corrected for 5 comparisons; i.e., each frequency band.

\begin{tabular}{ccccc|ccc}
\cline { 2 - 7 } & \multicolumn{4}{c|}{ RP Response vs. Baseline } & \multicolumn{3}{c}{$\Delta$} \\
\multicolumn{1}{c}{ Controls } & \multicolumn{2}{c}{ SCH } & \multicolumn{2}{c}{$\Delta$ RP Controls vs. SCH patients } \\
& $t[50]$ & $p$-value & $t[27]$ & $p$-value & $F[1,77]$ & $p$-value & partial $\eta^{2}$ \\
\hline$\theta$ & 10.217 & $<0.0001$ & 2.970 & 0.006 & 13.867 & $<0.0001$ & 0.153 \\
$\alpha$ & -11.194 & $<0.0001$ & -6.027 & $<0.0001$ & 14.574 & $<0.0001$ & 0.159 \\
$\beta_{1}$ & -13.986 & $<0.0001$ & -6.235 & $<0.0001$ & 11.701 & 0.001 & 0.132 \\
$\beta_{2}$ & -11.124 & $<0.0001$ & -4.530 & $<0.0001$ & 9.598 & 0.003 & 0.111 \\
$\gamma$ & -2.099 & 0.041 & -1.283 & 0.210 & 0.442 & 0.508 & 0.006 \\
\hline
\end{tabular}

Non-significant differences were observed for gamma. Between-group analyses for RP showed that mean $\triangle \mathrm{RP}$ values were statistically significantly lower for SCH patients than for controls in the theta band, whereas the opposite behavior was found for alpha, beta- 1 and beta- 2 . Non-significant effects were found in the gamma band. The results of both tests are displayed in table 3 . Figure $3 \mathrm{~b}$ summarizes the boxplots corresponding to the grand-average $\Delta \mathrm{RP}$ values for target tones.

It is interesting to point out that there seems to be a synchronized pattern between $\triangle K L D$ and $\triangle \mathrm{RP}$ in Figure 2 . In order to analyze the statistical dependence between $\triangle \mathrm{KLD}$ and $\triangle \mathrm{RP}$, the symmetric uncertainty was computed. The symmetric uncertainty is a normalized definition of the mutual information [36]. Figure 2 shows the symmetric uncertainty results (the larger the symmetric uncertainty, the more dependent one parameter is on the other). As can be observed in Figure 2, the symmetric uncertainty is equal to one for self-comparisons and is higher for comparisons between the same bands. However, these values are never greater than 0.46 , with a maximum value between $\triangle K L D$ and $\triangle \mathrm{RP}$ in the theta band. 


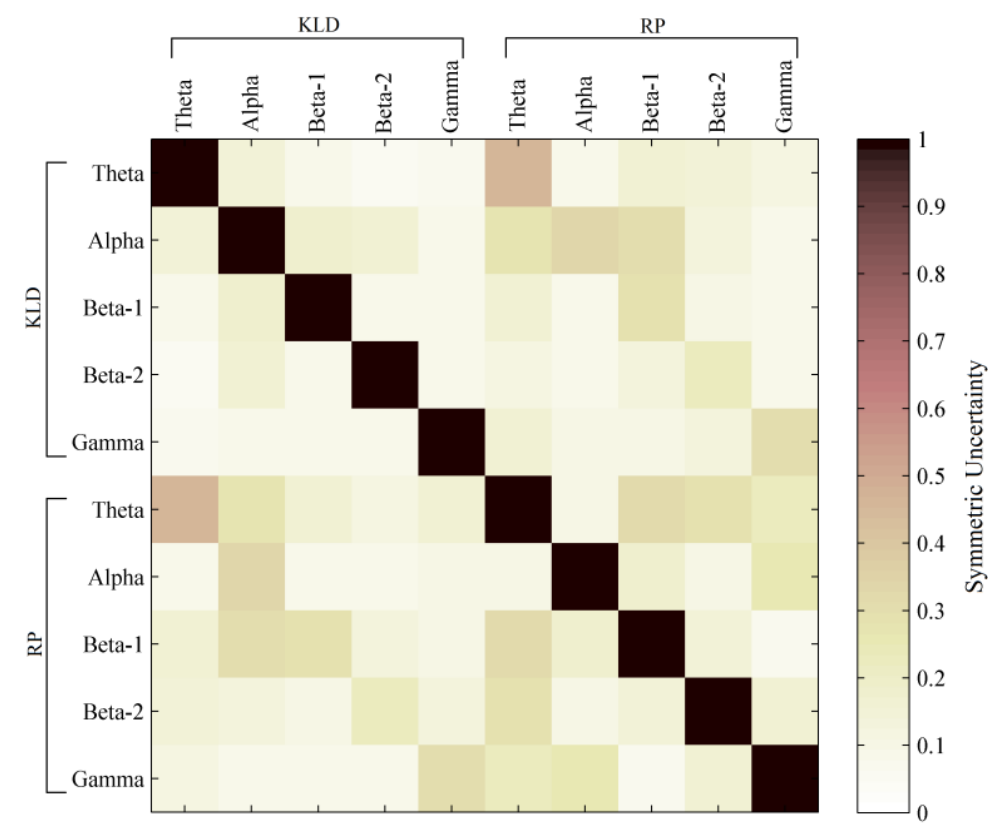

Figure 2. Map showing symmetric uncertainty between grand-average $\triangle K L D$ and $\triangle R P$ in different frequency bands.

\subsection{Sensor level analyses}

In a second step, KLD and $\triangle$ KLD spatial patterns were explored. Figure 4 shows the results of the dependent-sample $t$-tests between baseline and response values for each group, as well as the results of the univariate ANOVA between groups. Only the alpha and beta-2 bands are shown in both figures, as they were the only bands that showed significant differences in the global analysis. A widespread statistically significant KLD increase from the baseline to the response window for both groups in all frequency bands $(p<0.01)$ was found. This change is, however, more significant for healthy controls than for $\mathrm{SCH}$ patients. Regarding between-group analyses, there were statistically significant differences between groups in the beta-2 band, located in the left parieto-occipital region. The results of the univariate ANOVA for $\triangle$ KLD are shown in figure 5 . The analysis shows widespread significant between-group differences in the alpha band and in the left parieto-occipital region in the beta- 2 band.

\subsection{Classification analyses}

ROC curves were computed to assess whether $\triangle \mathrm{KLD}$ in conjunction with $\triangle \mathrm{RP}$ could discriminate $\mathrm{SCH}$ patients from controls by means of LR LOO-CV. In this method, each subject is excluded from the training set one at a time and then classified with the predictor built from the data of the other subjects [37]. Although classification statistics decrease with this procedure [38], it provides a nearly unbiased estimation of the true error rate [37]. Stepwise LR allows the selection of features that may not be significant by themselves but could provide a performance improvement when taken in conjunction with others. As previously explained, the LR was performed for each fold of the $\mathrm{CV}$ and the eight features that were selected the most were chosen for the final evaluation [35]. The eight features selected by the binary stepwise LR for each dataset are presented in table 4. The resulting ROC curves are summarized in figure 6, while the classification statistics are summarized in table 5. The feature selection procedure achieved higher classification statistics than the averaging of KLD values across channels. 

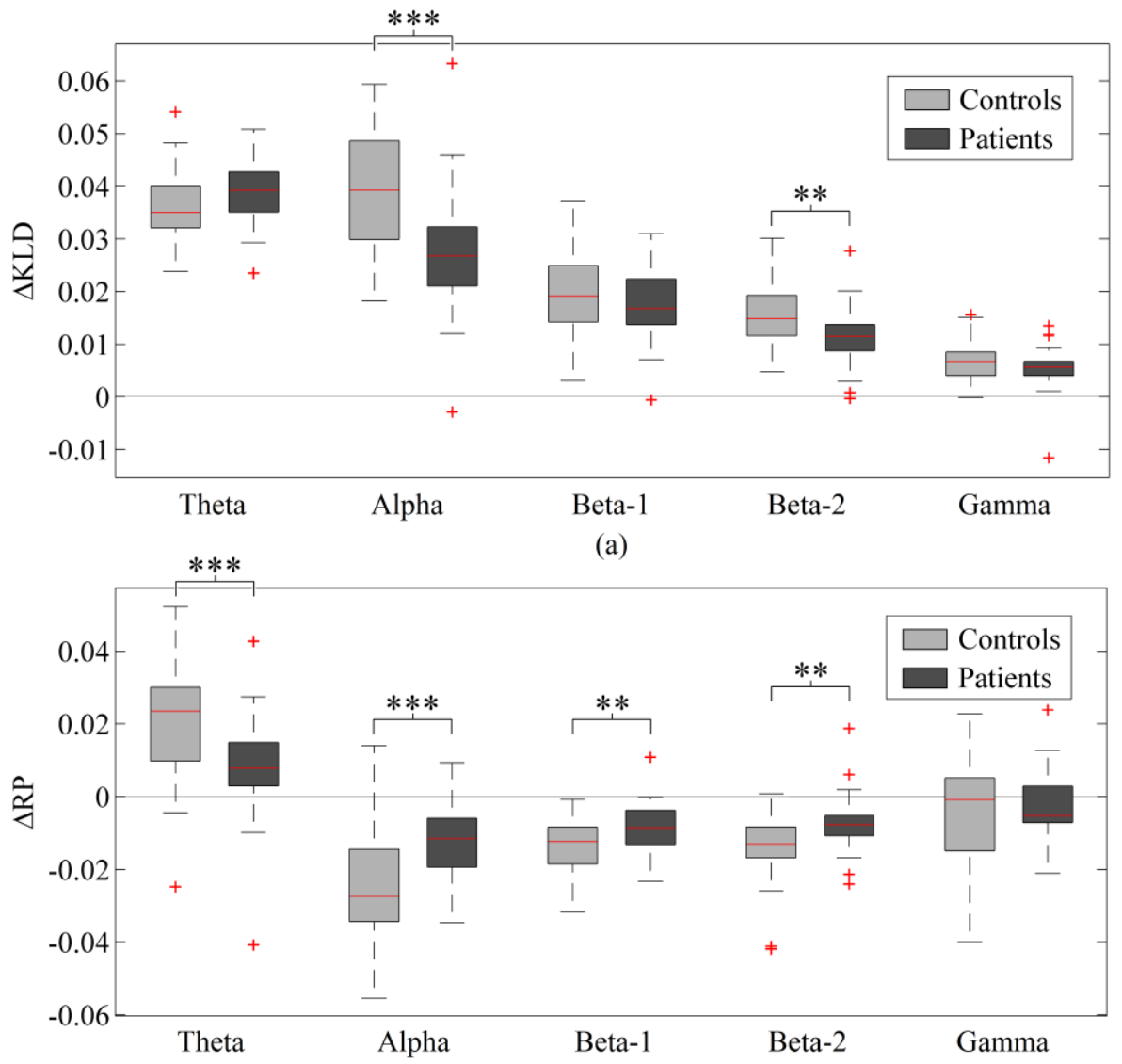

(b)

Figure 3. Boxplots depicting (a) grand-average $\triangle K L D$ values at each frequency band and (b) grandaverage $\triangle R P$ values at each frequency band. Positive values indicate an increase from the baseline to the response window, while negative values indicate a decrease. Statistically significant between-group differences are marked with asterisks: **, $\mathrm{p}<0.01$; ***, $\mathrm{p}<0.001$. Bonferroni corrected for 5 comparisons; i.e., each frequency band.

Table 4. Features selected by the binary stepwise LR.

\begin{tabular}{ccc}
\hline & Dataset & \\
\hline FS4 & FS2 & FS3 \\
\hline$\Delta$ KLD F7 Beta-2 & $\Delta$ KLD PO10 Theta & $\Delta$ RP O2 Theta \\
$\Delta$ KLD Fp1 Gamma & $\Delta$ KLD T7 Alpha & $\Delta$ RP PO9 Theta \\
$\Delta$ KLD Fp2 Gamma & $\Delta$ KLD P3 Beta-2 & $\Delta$ RP Oz Theta \\
$\Delta$ RP PO9 Theta & $\Delta$ KLD O1 Beta-2 & $\Delta$ RP Fp1 Alpha \\
$\Delta$ RP Oz Theta & $\Delta$ KLD FC6 Beta-2 & $\Delta$ RP Pz Alpha \\
$\Delta$ RP Fp1 Alpha & $\Delta$ KLD CP2 Beta-2 & $\Delta$ RP T8 Beta-1 \\
$\Delta$ RP Pz Alpha & $\Delta$ KLD Fp1 Gamma & $\Delta$ RP Fp1 Beta-2 \\
$\Delta$ RP Fp1 Beta-2 & $\Delta$ KLD Cz Gamma & $\Delta$ RP Fp1 Gamma \\
\hline
\end{tabular}




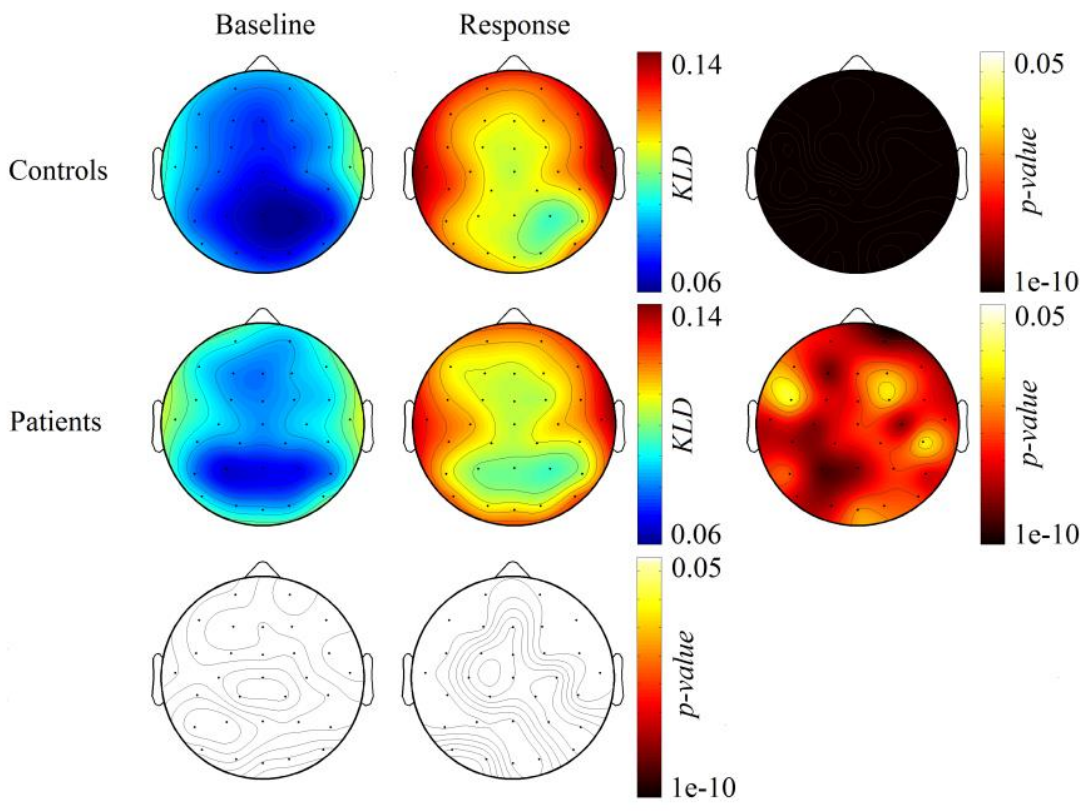

(a)



(b)

Figure 4. Topographic maps of the average KLD values and statistical comparisons from baseline to response at each electrode location in the (a) alpha band and (b) beta-2 band. The left column shows the average KLD values in the baseline window while the center column shows the average values in the response window. The right column displays the within-group differences between the baseline and response windows (dependent-samples $t$-tests with FDR correction), while the bottom row shows between-group differences for each window (univariate ANOVAs with FDR correction). FDR corrected for 31 comparisons; i.e., each EEG channel. 

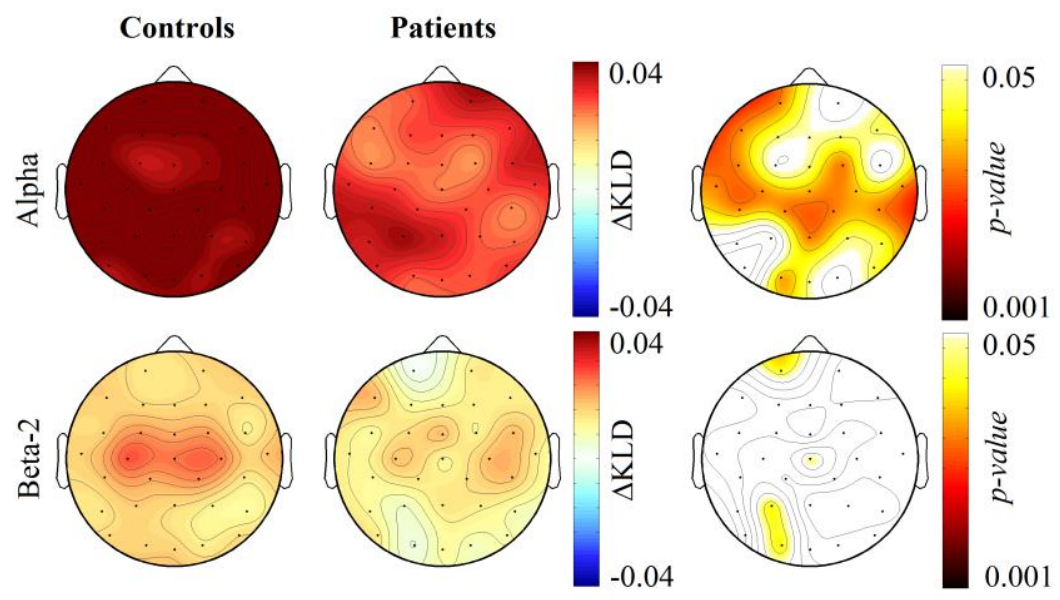

Figure 5. Topographic maps of the $\triangle \mathrm{KLD}$ values and statistical comparisons between groups at each electrode location. The left column shows the corrected $\triangle$ KLD values for controls, while the center column shows the corrected values for SCH patients. The right column summarizes the between-group statistical differences (univariate ANOVAs with FDR correction). FDR corrected for 31 comparisons; i.e., each EEG channel.



Figure 6. ROC curves for feature sets FS2, FS3 and FS4. The continuous gray line represents the ROC curve for the feature set formed by $\triangle K L D$ values at specific electrode locations and frequency bands, selected by a binary stepwise LR (FS2). The dashed line represents the ROC curve for the feature set formed by $\Delta R P$ values at specific electrode locations and frequency bands, selected by a binary stepwise LR (FS3). The continuous black line represents the ROC curve for the feature set formed by $\triangle$ KLD and $\Delta \mathrm{RP}$ values at specific electrode locations and frequency bands, selected by a binary stepwise LR (FS4).

Table 5. Classification statistics obtained for the LR LOO-CV.

\begin{tabular}{lcccc}
\cline { 2 - 5 } & \multicolumn{4}{c}{ Feature sets } \\
\cline { 2 - 5 } & FS1 & FS2 & FS3 & FS4 \\
\hline Sensitivity & 0.5714 & 0.6786 & 0.7857 & 0.8929 \\
Specificity & 0.9020 & 0.8627 & 0.9216 & 0.9020 \\
Accuracy & 0.7848 & 0.7975 & 0.8734 & 0.8987 \\
AROC & 0.7878 & 0.8817 & 0.9160 & 0.9510 \\
\hline
\end{tabular}




\section{Discussion}

The aim of this study was to explore non-stationarity patterns during an auditory oddball task in SCH by means of KLD, check classification performance and assess the complementarity of KLD with RP. To this end, three main objectives were set.

\subsection{Characterization of neural reorganization}

The first objective was to assess whether KLD could characterize neural reorganization during an auditory oddball task. Both groups showed widespread significant changes in KLD nonstationarity from the baseline to the response window in all frequency bands. However, this change was lower in SCH patients in all frequency bands, except theta. This seems to suggest less widespread neural reorganization in response to relevant stimuli for SCH patients. The widespread changes in KLD also confirm that it can indeed characterize neural reorganization. Other studies have reported similar results with different measures. Bachiller et al. [12] found a global decrease of Shannon entropy and median frequency in controls in response to target stimuli that was also observed in SCH patients as well. SCH patients, however, showed less statistically significant changes than controls, located around Pz. On the other hand, significant median frequency changes were almost as widespread in $\mathrm{SCH}$ patients as in controls [12]. This study, however, only measured global changes, as opposed to evaluating changes in every frequency band.

The statistically significant smaller change in non-stationarity in SCH patients with respect to controls during a target tone in the alpha and beta- 2 bands could also suggest a decreased response to novelty and salience in $\mathrm{SCH}$. This would be in agreement with the theory of aberrant salience detection in $\mathrm{SCH}$ [39], as this mental disorder could manifest itself as a decreased response to relevant stimuli during the auditory oddball task [40]. It has been suggested that $\mathrm{SCH}$ patients show abnormal activation in response to attention tasks [40]. This may be related to the hyperactivation of the default mode network (regions of the brain more active during rest than during cognitive tasks) that has been reported in $\mathrm{SCH}$ patients [41].

To analyze whether the present RP results can replicate the findings obtained with a different database, we compared the results with a previous study [15]. In the aforementioned study, $\Delta \mathrm{RP}$ was positive in the theta band for both SCH patients and controls, with the increase being lower for patients. These results agree with the present study, where controls show higher $\Delta \mathrm{RP}$ in the theta band than SCH patients. $\triangle \mathrm{RP}$ was negative in the alpha band, which also coincides with our findings, where both groups exhibit a decrease, but it is more pronounced for controls than for patients. There was also a widespread RP decrease for high frequencies in controls that is also present in our study. Overall, we can conclude that the global patterns are similar in both studies. The symmetric uncertainty results suggest that $\triangle K L D$ and $\triangle \mathrm{RP}$ might be related to some extent. However, these values are never greater than 0.46 , which indicates that the measures provide different information. This is further supported by the classification results discussed in section 5.3.

Regarding the comparison among standard, distractor and target tones, the repeated measures ANOVA showed that target tones display the most significant 'group' by 'window' by 'frequency band' interactions among all tone types followed by distractor tones and finally standard tones. It is noteworthy that as tones become novel (distractor tones) and both novel and relevant (target) the interactions between factors become more significant. This supports the notion that non-stationarity is affected by the processing of relevant and novel information and could be related to the aberrant salience hypothesis, according to which $\mathrm{SCH}$ patients show abnormal activation in response to attentional tasks [40].

\subsection{Non-stationarity differences between groups}

After comparing the baseline-corrected response values, statistically significant differences were found in the alpha and beta- 2 bands. These results support the notion that $\mathrm{SCH}$ patients are not 
able to reconfigure their brain activity during a cognitive task. In particular, the alpha band showed widespread significant abnormalities.

ERP studies have found that different brain systems are related to task-related activation to targets (top-down processing) and inhibition of response to irrelevant distracting events (bottom-up processing) [40]. Alpha activity has been associated with functional inhibition of task irrelevant processing during internal tasks [42], as well as with the long-distance coordination of gamma oscillations [43,44]. However, recent research has shown that alpha activity plays an active role in information processing, and that transient change in alpha activity by means of external stimuli leads to alterations in cognitive performance [42]. A topdown process is an attentional control function that keeps processes focused on task performance by inhibiting interference from brain areas irrelevant to the task [42]. Top-down control is more relevant in tasks that need internal processing, as opposed to the processing of sensory information, which is guided by bottom-up processes [42]. An auditory-oddball task demands the full attention of participants, as they must focus on relevant tones and press a button when they hear them, while ignoring non-relevant tones. Consequently, the increase in alpha non-stationarity could be interpreted in terms of top-down control during an attentiondemanding task. This further challenges the traditional belief that alpha is only associated with inhibition of response to irrelevant events [42]. It has been suggested that $\mathrm{SCH}$ patients show impairment in cognitive performance [45] and in this study they show less alpha nonstationarity compared to controls. Moreover, a previous study found event-related alpha attenuation in SCH patients compared to healthy controls, exclusively at the posterior temporal regions [46].

Beta oscillations have been associated with sensory-motor coordination [8]. This function is relevant because in the auditory oddball task participants must press a button when they hear a target tone. In our study, significant $\triangle \mathrm{KLD}$ differences were found between groups in beta-2, with $\triangle K L D$ values being higher for controls in the left parieto-occipital region. This supports the notion that sensory-motor coordination could be impaired in SCH patients. In a previous study [7] it was found that $\mathrm{SCH}$ patients were not able to change their neural coupling patterns from baseline to response in the beta- 2 band, which could be in agreement with our results. It is possible that the $\triangle \mathrm{KLD}$ difference between controls and patients in the beta- 2 band is related to a hyperactive baseline state in $\mathrm{SCH}$ patients [5]. There were significant between-group differences in the baseline window in the beta-2 band, which were located in the same region as the $\triangle$ KLD differences (left parieto-occipital). Consequently, the $\triangle$ KLD differences are due to $\mathrm{SCH}$ patients showing more non-stationarity in the baseline window than patients, rather than controls showing more non-stationarity in the response window. During task performance, activation in the default network decreases for healthy subjects [41]. Previous studies found that SCH patients showed hyperactivation of the default network during and auditory oddball and working memory tasks [5,41]. Our results further support the notion that this hyperactivation may be involved in the core cognitive symptoms of SCH [41]. It is important to point out, however, that behavioral differences can have an influence on sensorimotor activity and thus in the statistical differences observed. The effect these differences might have on the observed results cannot be neglected.

\subsection{Classification performance}

Regarding KLD classification performance, our results showed that using the average $\triangle$ KLD values at each frequency band as features gave the lowest performance results. On the other hand, the application of a feature selection procedure to select $\triangle \mathrm{KLD}$ and $\triangle \mathrm{RP}$ channels significantly increased classification statistics. The features selected by this procedure obtained an accuracy of $89.87 \%$, sensitivity of $89.29 \%$, specificity of $90.20 \%$ and area under ROC (AROC) of 0.9510 . The obtained AROC was greater when jointly selecting $\triangle \mathrm{KLD}$ and $\triangle \mathrm{RP}$ features than when only $\triangle \mathrm{KLD}$ or $\triangle \mathrm{RP}$ features were used. These results suggest that $\triangle \mathrm{KLD}$ in conjunction with $\triangle \mathrm{RP}$ might be able to differentiate EEG activity between SCH patients and healthy controls and that these two measures provide different information. 
Other studies have attempted to classify SCH patients and controls with EEG measures. For example, Sabeti et al. [47] reported a classification accuracy of $89 \%$ and $91 \%$ with linear discriminant analysis and Adaboost, respectively. They computed Shannon entropy, spectral entropy, approximate entropy, Lempel-Ziv complexity and Higuchi fractal dimension for 20 SCH patients and 20 healthy subjects, in 20 electrode locations. After this, they applied a feature selection procedure based on genetic programming [47] and obtained classification statistics with LOO-CV. It is noteworthy that the EEG recordings were not acquired during an auditory oddball task and instead were 2-minute length eyes-open recordings and there were also fewer subjects under study. In another study, SCH classification was attempted with magnetoencephalographic (MEG) recordings [48]. The subject group consisted of a total of 15 SCH patients and 17 controls. Two feature sets, with and without feature selection were used for classification. The features were median frequency, spectral entropy and relative power and performance was evaluated with LR LOO-CV. This study obtained an accuracy of $71.3 \%$, sensitivity of $68.0 \%$, specificity of $74.2 \%$ and AROC of 0.74 for the feature selection set. The MEG recordings were 5-minute length eyes-closed recordings [48]. Overall, our results provide similar classification accuracy to those of the aforementioned studies, while improving its robustness. This may suggest that $\triangle \mathrm{KLD}$ and $\triangle \mathrm{RP}$ are two complementary measures that can be useful to discriminate between healthy subjects and $\mathrm{SCH}$ patients.

\subsection{Limitations and future research lines}

There are certain limitations in our study that merit further attention. The number of patients should be increased to bring it closer to the number of control subjects. In particular, classification results were satisfactory but there needs to be a large population so that separate training and test groups can be formed. More reliable classification statistics could be obtained this way. It is also worth mentioning that the treatment for SCH patients can have an effect on the statistical differences between groups. In order to study this bias, it would be interesting to include another group composed of SCH patients that have not received any previous treatment. To be able to study the delta band, the acquisition protocol should be changed to significantly increase the window length, since the response window must contain a minimum number of oscillation periods [7]. In this study, KLD was used to quantify how much the TFR differs from a uniform distribution for both the baseline and response windows. However, as KLD allows the comparison between any pair of distributions, it would be interesting to measure the divergence between the TFR in the response and baseline windows. In addition, it could be interesting to study other non-stationarity measures that could also characterize neural reorganization and provide further information on neural dynamics in SCH. Finally, further studies should analyze neural reorganization at the source level, which could give a more complete characterization of neural coupling.

\section{Conclusions}

KLD is a measure of non-stationarity that can characterize neural dynamics during active attention tasks in different frequency bands, allowing researchers to focus on different parts of the spectrum and their individual dynamics in the baseline and response windows. Our findings support the idea that $\mathrm{SCH}$ is associated with a decreased response to novelty and salience, as well as a decreased reconfiguration of brain activity during a cognitive task. Abnormalities in neural reorganization were found in $\mathrm{SCH}$ patients during an auditory oddball task compared to healthy controls. These abnormalities were mostly found in the alpha band, which may support the notion that this band is involved in information processing. Furthermore, we found that the differences in $\triangle$ KLD in the beta- 2 band were caused by SCH patients showing hyperactivation in the baseline window. A feature selection procedure suggested that KLD and RP can be complementary measures. Moreover, the classification statistics obtained indicate that KLD in conjunction with RP could be a promising candidate to delimitate potential SCH biomarkers. 


\section{Acknowledgements}

This research project was supported in part by the projects TEC2014-53196-R of 'Ministerio de Economía y Competitividad' and FEDER; the project VA037U16 from the "Consejería de Educación de la Junta de Castilla y León", the "Fondo de Investigaciones Sanitarias (Instituto de Salud Carlos III)" under projects FIS PI11/02203 and PI15/00299; and the "Gerencia Regional de Salud de Castilla y León" under projects GRS 932/A/14 and GRS 1134/A/15. P. Núñez was in receipt of a 'Promoción de empleo joven e implantación de la Garantía Juvenil en I $+\mathrm{D}+\mathrm{i}$ ' grant from 'Ministerio de Economía y Competitividad' and the University of Valladolid, A. Bachiller and J. Gomez-Pilar were in receipt of a PIF-UVA grant from the University of Valladolid. A. Lubeiro has a predoctoral scholarship from the "Junta de Castilla y León" and European Social Fund.

\section{References}

[1] Saha S, Chant D, Welham J and McGrath J 2005 A systematic review of the prevalence of schizophrenia PLoS Med. 2 0413-33

[2] McGrath J, Saha S, Chant D and Welham J 2008 Schizophrenia: A concise overview of incidence, prevalence, and mortality Epidemiol. Rev. 30 67-76

[3] Knapp M 2000 Schizophrenia costs and treatment cost-effectiveness Acta Psychiatr. Scand. Suppl. $10215-8$

[4] Roach B J and Mathalon D H 2008 Event-related EEG time-frequency analysis: An overview of measures and an analysis of early gamma band phase locking in schizophrenia Schizophr. Bull. 34 $907-26$

[5] Molina V, Bachiller A, Suazo V, Lubeiro A, Poza J and Hornero R 2014 Noise power associated with decreased task-induced variability of brain electrical activity in schizophrenia Eur. Arch. Psychiatry Clin. Neurosci. 1-7

[6] Gomez-Pilar J, Poza J, Bachiller A, Gómez C, Molina V and Hornero R 2015 Neural network reorganization analysis during an auditory oddball task in schizophrenia using wavelet entropy Entropy 17 5241-56

[7] Bachiller A, Poza J, Gómez C, Molina V, Suazo V and Hornero R 2015 A comparative study of event-related coupling patterns during an auditory oddball task in schizophrenia J. Neural Eng. 12 16007

[8] Uhlhaas P J, Haenschel C, Nikolić D and Singer W 2008 The role of oscillations and synchrony in cortical networks and their putative relevance for the pathophysiology of schizophrenia Schizophr. Bull. 34 927-43

[9] Makeig S, Debener S, Onton J and Delorme A 2004 Mining event-related brain dynamics Trends Cogn. Sci. 8 204-10

[10] Le Van Quyen M, Foucher J, Lachaux J, Rodriguez E, Lutz A, Martinerie J and Varela F J 2001 Comparison of Hilbert transform and wavelet methods for the analysis of neuronal synchrony. $J$. Neurosci. Methods 111 83-98

[11] Ford J M, Roach B J, Hoffman R S and Mathalon D H 2005 The dependence of P300 amplitude on gamma synchrony breaks down in schizophrenia Biophys. Chem. 257 2432-7

[12] Bachiller A, Lubeiro A, Díez A, Suazo V, Domínguez C, Blanco J A, Ayuso M, Hornero R, Poza J and Molina V 2015 Decreased entropy modulation of EEG response to novelty and relevance in schizophrenia during a P300 task Eur. Arch. Psychiatry Clin. Neurosci. 265 525-35

[13] Bassett D S, Nelson B G, Mueller B A, Camchong J and Lim K O 2012 Altered resting state complexity in schizophrenia Neuroimage 59 2196-207

[14] Takahashi T, Cho R Y, Mizuno T, Kikuchi M, Murata T, Takahashi K and Wada Y 2010 Antipsychotics reverse abnormal EEG complexity in drug-naive schizophrenia : a multiscale entropy analysis Neuroimage $\mathbf{5 1} 173-82$

[15] Bachiller A, Díez A, Suazo V, Domínguez C, Ayuso M, Hornero R, Poza J and Molina V 2014 Decreased spectral entropy modulation in patients with schizophrenia during a P300 task Eur. Arch. Psychiatry Clin. Neurosci. 264 533-43

[16] Blanco S, Garcia H, Quiroga R Q, Romanelli L and Rosso O A 1995 Stationarity of the EEG series IEEE Eng. Med. Biol. Mag. 14 395-9

[17] Nason G P 2006 Stationary and non-stationary time series Stat. Volcanol. 129-43

[18] Stoica P, Moses R L and Hall P 2005 Introduction to spectral analysis Technometrics 47 104-5

[19] Tong S, Li Z, Zhu Y and Thakor N V 2007 Describing the nonstationarity level of neurological signals based on quantifications of time-frequency representation IEEE Trans. Biomed. Eng. 54 
$1780-5$

[20] Rioul O and Vetterli M 1991 Wavelets and Signal Processing IEEE Signal Process. Mag. 8 14-38

[21] Núñez P, Poza J, Gomez-Pilar J, Bachiller A, Gómez C, Lubeiro A, Molina V and Hornero R 2016 Analysis of the non-stationarity of neural activity during an auditory oddball task in schizophrenia Proc. of the 35th Annual Int. Conf. of the IEEE Engineering in Medicine and Biology Society Conference (Orlando, FL, 2016) pp 3724-7

[22] Basseville M 1989 Distance measures for signal processing and pattern recognition Signal Processing 18 349-369

[23] Weinberg G V. 2016 Kullback-Leibler divergence and the Pareto-Exponential approximation Springerplus 5604

[24] Quian Quiroga R, Arnhold J, Lehnertz K and Grassberger P 2000 Kulback-Leibler and renormalized entropies: Applications to electroencephalograms of epilepsy patients Phys. Rev. E - Stat. Physics, Plasmas, Fluids, Relat. Interdiscip. Top. 62 8380-6

[25] American Psychiatric Association 2013 Diagnostic and Statistical Manual of Mental Disorders, 5th Edition (Arlington, VA: American Psychiatric Publishing)

[26] Goncharova I I, McFarland D J, Vaughan T M and Wolpaw J R 2003 EMG contamination of EEG: Spectral and topographical characteristics Clin. Neurophysiol. 114 1580-93

[27] Bledowski C, Prvulovic D, Hoechstetter K, Scherg M, Wibral M, Goebel R and Linden D E J 2004 Localizing P300 generators in visual target and distractor processing: a combined event-related potential and functional magnetic resonance imaging study J. Neurosci. 24 9353-60

[28] Tallon-Baudry C, Bertrand O, Delpuech C and Pernier J 1996 Stimulus specificity of phase-locked and non-phase-locked $40 \mathrm{~Hz}$ visual responses in human J. Neurosci. 16 4240-9

[29] Samar V J, Bopardikar A, Rao R and Swartz K 1999 Wavelet analysis of neuroelectric waveforms: a conceptual tutorial Brain Lang. 66 7-60

[30] Mallat S 1999 A Wavelet Tour of Signal Processing (San Diego: Academic Press)

[31] Torrence C and Compo G P 1998 A practical guide to wavelet analysis Bull. Am. Meteorol. Soc. 79 61-78

[32] Poza J, Gómez C, Bachiller A and Hornero R 2012 Spectral and non-linear analyses of spontaneous magnetoencephalographic activity in alzheimer's disease J. Healthc. Eng. 3 299-322

[33] Benjamini Y and Hochberg Y 1995 Controlling the false discovery rate: a practical and powerful approach to multiple testing J. R. Stat. Soc. 57 289-300

[34] Bender R and Lange S 1999 Multiple test procedures other than Bonferroni's deserve wider use. BMJ 318 600-1

[35] Guyon I and Elisseeff a 2003 An introduction to variable and feature selection J. Mach. Learn. Res. 3 1157-82

[36] Särndal C E 1974 A comparative study of association measures Psychometrika 39 165-87

[37] Simon R, Radmacher M D, Dobbin K and Mcshane L M 2003 Pitfalls in the use of DNA microarray data for diagnostic and prognostic classification J. Natl. Cancer Inst. 95 14-8

[38] Gómez C, Hornero R, Abásolo D, Fernández A and Escudero J 2009 Analysis of MEG background activity in Alzheimer's disease using nonlinear Methods and ANFIS Ann. Biomed. Eng. 37 586-94

[39] Kapur S 2003 Psychosis as a state of aberrant salience: A framework linking biology, phenomenology, and pharmacology in schizophrenia Am. J. Psychiatry 160 13-23

[40] Gur R E, Turetsky B I, Loughead J, Snyder W, Kohler C, Elliott M, Pratiwadi R, Ragland J D, Bilker W B, Siegel S J, Kanes S J, Arnold S E and Gur R C 2007 Visual attention circuitry in schizophrenia investigated with oddball event-related functional magnetic resonance imaging Am. $J$. Psychiatry 164 442-9

[41] Whitfield-Gabrieli S, Thermenos H W, Milanovic S, Tsuang M T, Faraone S V, McCarley R W, Shenton M E, Green A I, Nieto-Castanon A, LaViolette P, Wojcik J, Gabrieli J D E and Seidman L J 2009 Hyperactivity and hyperconnectivity of the default network in schizophrenia and in firstdegree relatives of persons with schizophrenia. Proc. Natl. Acad. Sci. U. S. A. 106 1279-84

[42] Klimesch W, Sauseng P and Hanslmayr S 2007 EEG alpha oscillations: The inhibition-timing hypothesis Brain Res. Rev. 53 63-88

[43] Palva S and Palva J M 2007 New vistas for $\alpha$-frequency band oscillations Trends Neurosci. 30 1508

[44] Jensen O, Bonnefond M and VanRullen R 2012 An oscillatory mechanism for prioritizing salient unattended stimuli Trends Cogn. Sci. 16 200-5

[45] Friston K J 1998 The disconnection hypothesis Schizophr. Res. 30 115-25

[46] Higashima M, Tsukada T, Nagasawa T, Oka T, Okamoto T, Okamoto Y and Koshino Y 2007 Reduction in event-related alpha attenuation during performance of an auditory oddball task in schizophrenia Int. J. Psychophysiol. 65 95-102 
[47] Sabeti M, Katebi S and Boostani R 2009 Entropy and complexity measures for EEG signal classification of schizophrenic and control participants Artif. Intell. Med. 47 263-74

[48] Escudero J, Ifeachor E, Fernández A, López-Ibor J J and Hornero R 2013 Changes in the MEG background activity in patients with positive symptoms of schizophrenia: spectral analysis and impact of age Physiol. Meas. 34 265-79 